focuses on topology issues to enforce emergency information sharing networks and to make relations between pertinent national and international agencies sustainable.

Methods: An agent-based simulation model with competitive and collaborative agents was applied to perform a simple, quantitative analysis of interagency and international cooperation networks with diverse topologies. An essential distinction of the information network topologies of the entities was detailed. The fact that all the agents of an entity are not only in collaboration, but in competition as well-vertically (for agents of adjacent levels) and horizontally (for agents of the same level) was implied. It was assumed that interagency and international interaction is horizontal only. To collate diverse topologies, the parameters characterizing the network efficiency and robustness such as flows, capacities, centrality, and relative size of the largest cluster were used.

Results: The optimal topology for interagency and international interaction prefers top-rank links for "tree"(hierarchical) networks and corresponds with middle-rank horizontal links in other cases.

Conclusions: It is of great value to support top-level contacts and ties of middle rank agents of national and international agencies for effective counteracting to global threats. Keywords: collaboration; disaster management; global threat; management

Prehosp Disaster Med

\section{Civil Defense Directives to Hospitals in Israel}

Maj. Aviv Obana, RN, MHA; Col. Ariel Bar, MD, MHA

Home Front Command, Medical Department, Israel

The directives for civil protection (Civil Defense) provided by the Home Front Command to hospitals in emergencies are vital to their uninterrupted function. These directives have to ensure the protection of the hospitalized patients and the hospital's staff as well as the continued functioning of the medical system.

Hospitals are complex organizations with needs and requirements not observed in other institutions. For example, patients are bedridden-they are dependent on fixed medical equipment and medical procedures, such as surgery or hemodialysis, that are being performed and cannot be discontinued during an alarm. Therefore, without pre-planned steps and preparedness, civil protection measures cannot be implemented immediately after the sounding of an alarm, except for mobile patients and visitors. These preparations include the transfer of medical wards to at least partially protected areas, the reduction of beds, and the evacuation of unprotected buildings and the upper floors of the hospital.

During national states of emergency in a total war, hospitals will have to function continuously, at full capacity, treating civilian and military casualties. Despite this, the experience in recent armed conflicts, such as the $2^{\text {nd }}$ Lebanon War and Operation Cast Lead, hospitals were not utilized to their capacity. During these conflicts, a number of hospitals were under missile bombardment, while only few numbers of casualties were admitted from the front line and the rear zones of the country.
In Operation Cast Lead, an internal re-organization in hospitals near the front line provided improved defense, but decreased the number of hospital beds in the Soroka Medical Center by $80 \%$ and by $30 \%$ in the Barzilai Hospital. New protection policies of hospitals during emergencies are defined by the optimal utilization of the hospitals' capacities, including maximum protection of patients and staff.

The principles of such policies include the vacation of beds, internal reorganization, and transfer of essential hospital wards to protected areas in order to ensure continued work and functioning. Such a re-organization was carried out during the $2^{\text {nd }}$ Lebanon War and Operation Cast Lead in hospitals. The new instructions for the protection of hospitals provide uniform directives, confirming with the states of preparedness within three defined levels: (1) Level Ahospitals are not in a threatened area and continue to function in a regular emergency mode; (2) Level B-hospitals are located in a threatened area, but the number of casualties is low, reorganization will provide improved security to patients and staff; and (3) Level $\mathrm{C}$-hospitals are under direct threat and the number of casualties is high. Changes are limited, and influenced mainly by hospitalization needs. Keywords: civil protection; emergency; hospitalization; hospitals; medical system; reorganization

Prehosp Disaster Med

\section{A Unique Tertiary Medicine Model for Israeli Defense Forces Soldiers}

Dorit Tekes-Manova; Avi Abargel; Jacob Habib

Medical Services and Supply Center, Israeli Defense Forces, Israel

Introduction: In Israel, there are no military hospitals. Therefore, tertiary care for ill or wounded soldiers is administrated exclusively by civilian hospitals. Nevertheless, the Israeli Defense Forces (IDF) remains responsible for the health and welfare of hospitalized soldiers, and the Medical Corps is informed and involved in their management.

In order to assure this coordination between the civilian centers and the Medical Corps, there are special army medical units are located within civilian hospitals. These units, which originally were established as registration units for hospitalized soldiers, are called "Yakar units" (meaning precious in Hebrew). The "Yakar units" have evolved, and currently provide various medical, military, and welfare services for hospitalized soldiers and their families.

Methods: The various roles of the army medical units in the Israeli 24 civilian medical centers were evaluated, including: (1) registering and controlling hospitalized and outpatient personnel; (2) facilitating coordination between the hospital and the army in every necessary aspect; (3) helping hospitalized soldiers connect with their families; (4) providing medical follow-up for hospitalized soldiers and managing their cases after discharge; (5) initiating medical counseling and conveying specialists when needed; (6) transferring soldiers from one hospital to another according to medical specialties or due to social reasons (convenience or request of the soldier and his family); (7) overseeing the welfare of the patients (bedside television, daily newspaper, transportation, etc.); (8) caring for and financing the needs 
of their families (accommodation, transportation, etc.); (9) transporting patients to rehabilitation centers; and (10) connecting the soldier with the Division of Rehabilitation in the Ministry of Defense.

Conclusions: This unique model enables coordination and collaboration between the army and the civilian hospitals. It ensures optimal treatment for sick and injured soldiers, and plays a crucial role during war and emergencies. Keywords: civil-military; hospital; Israel; military; tertiary care Prebosp Disaster Med

The Federal Bureau of Investigation/Centers for Disease Control and Prevention Joint Criminal and Epidemiological Investigations Course: Enhancing Relationships to Improve Biothreat Readiness

K.E. Hayashi; S.S. Papagiotas

US Centers for Disease Control and Prevention, Atlanta, Georgia USA

Introduction: Bioterrorism incidents necessitate that law enforcement and public health communities coordinate criminal and epidemiological investigations to protect public health and safety. Since these entinties may not interact routinely, information sharing and investigation coordination can be challenging. To facilitate inter-agency communication, the (US) Centers for Disease Control and Prevention $(\mathrm{CDC})$ and the Federal Bureau of Investigation (FBI) developed a Joint Criminal and Epidemiological Investigations Course for public health and law enforcement personnel. The course is designed to develop relationships and promote information exchange between public health and law enforcement.

Methods: The joint investigations course is a two-day curriculum of lectures and exercises that is facilitated by CDC and FBI instructors. Lectures provide an overview of investigative methods and information sharing practices while the exercises reinforce the principles and techniques highlighted in the lectures.

Results: Since 2004, the Joint Investigations Course has been conducted 13 times, training $>800$ students. Since there has not been a major bioterrorism incident in the US since 2001, it is difficult to determine if there is significantly increased information sharing and collaboration between public health and law enforcement. Course evaluations indicated that the training has sometimes served as the first opportunity for public health and law enforcement interaction in a jurisdiction. Additionally, it has been reported that jurisdictions that have completed the course have used joint investigation methods to respond successfully to incidents involving threat agents.

Conclusions: The course is considered an effective method for developing essential linkages between law enforcement and public health in responding to bioterrorism. While the course initially was developed in the US, it may be a useful model for use by other countries.

Keywords: bioterrorism; Centers for Disease Control and Prevention; criminal; epidemiology; Federal Bureau of Investigation; investigation

Prehosp Disaster Med
Threat of Ambulance Car Bombs Arriving at Hospitals and Other Mass-Casualty Scenes-Intelligence Review and Methods for Mitigation

Reuven Keren; Mr. David Shwartz

Israeli Ministry of Health Security Division, Israel

Introduction: Hospitals are no longer safe havens, as they can be alluring targets to terrorists in various parts of the world. Among possible modes of operation, one modus operandi stood out-terrorist groups plan and attempt to infiltrate hospitals using ambulances that are transporting trauma patients from scenes of mass casualties. The threat of terrorists arriving at the emergency department requires measures to counter such a possibility, without the medical condition of the evacuees deteriorating.

Methods: Researchers consulted with trauma specialists and wrote new standard operating procedures (SOPs) regarding the security clearance of ambulances, which were verified by the Israeli Police and Ministry of Health. They also trained the security staff and implemented the plan.

Results: In the years since the implementation of the new SOPs, and through a multitude of terrorist attacks and other national emergencies, thousands of ambulances were screened by security guards at the entrances to Israeli hospitals. In none of the cases, has a victim or medical staff member claimed that the medical condition deteriorated due to these tighter security measures.

Conclusions: Altough stopping or delaying ambulances conveying victims with trauma wounds can deteriorate the medical condition of the victims, allowing the uninterrupted approach of ambulances into the hospitals poses an imminent hazard.

Based on the results of this SOP, delaying an ambulance for 30-40 seconds has no negative effect on the victims. Yet, the security screening nullifies the possibility of infiltration by this modus operandi, even during mass-casualty incidents, when a large number of ambulances are arriving at the hospital.

Keywords: ambulance; car bomb; hospital; mass-casualty incident; standard operating procedure; terrorism

Prebosp Disaster Med

\section{The Israeli Committee on Conventional Mass Casualty Situations \\ Mosbe Micbaelson, $M D$}

Head of the Israeli Committee on Conventional Mass Casualty Situations, Israel

The Israeli Committee on Conventional Mass Casualty Situations (ICCMCS) has been a part of the Emergency Division of the Ministry of Health for $>30$ years. Its members are appointed by the General Director of the Ministry of Health. Members include doctors, nurses, health administrators, and representatives of the Pre-Hospital Forces.

The committee advises the Minister of Health on the guidelines for dealing with Conventional MCS. With the approval of the Ministry of Health, these guidelines are distributed to all hospitals, where local committees transform them to written standing orders that are returned to the Emergency Division for approval.

As the state of Israel has experienced a number of MCSs, experiences are included in the guidelines. Thus, 